\title{
CALDERÓN-LOZANOVSKIĬ CONSTRUCTION FOR MIXED NORM SPACES
}

\author{
L. MALIGRANDA* (Luleå)
}

\begin{abstract}
We show that the Calderón-Lozanovskiu construction $\varphi(\cdot)$ commutes with arbitrary mixed norm spaces, that is,

$$
\varphi\left(E_{0}\left[F_{0}\right], E_{1}\left[F_{1}\right]\right)=\varphi\left(E_{0}, E_{1}\right)\left[\varphi\left(F_{0}, F_{1}\right)\right]
$$

if and only if $\varphi$ is equivalent to a power function. This result we obtain by giving characterizations of the corresponding embeddings of $\varphi\left(E_{0}\left[F_{0}\right], E_{1}\left[F_{1}\right]\right)$ into $\varphi_{0}\left(E_{0}, E_{1}\right)\left[\varphi_{1}\left(F_{0}, F_{1}\right)\right]$ and vice versa in terms of the functions $\varphi, \varphi_{0}, \varphi_{1}$. As a particular case, we get embeddings of an Orlicz space with mixed norms into an Orlicz space on a product of measure spaces. Applications to classical operators between mixed norm Orlicz spaces are also discussed.
\end{abstract}

\section{Introduction}

For vector-valued $L_{p}$-spaces the following Lions-Peetre formula is known (see $[2])$ :

$$
\left(L_{p_{0}}\left(X_{0}\right), L_{p_{1}}\left(X_{1}\right)\right)_{\theta, p}=L_{p}\left(\left(X_{0}, X_{1}\right)_{\theta, p}\right),
$$

and also the Calderón result (see [10], [2])

$$
\left[L_{p_{0}}\left(X_{0}\right), L_{p_{1}}\left(X_{1}\right)\right]_{\theta}=L_{p}\left(\left[X_{0}, X_{1}\right]_{\theta}\right),
$$

where $1 \leqq p_{0}, p_{1}<\infty$ and $1 / p=(1-\theta) / p_{0}+\theta / p_{1}, 0<\theta<1$. Calderón [10] proved this result even for general vector-valued spaces. He showed that

$$
\left[E_{0}\left(X_{0}\right), E_{1}\left(X_{1}\right)\right]_{\theta} \hookrightarrow E_{0}^{\theta} E_{1}^{1-\theta}\left(\left[X_{0}, X_{1}\right]_{\theta}\right),
$$

\footnotetext{
* Research supported by the Swedish Natural Science Research Council (NFR)-grant M5105$20005228 / 2000$.

Key words and phrases: Banach ideal spaces, Banach function lattices, Calderón-Lozanovskiǔ spaces, Orlicz spaces, mixed norm spaces, mixed norm $L_{p}$-spaces, mixed norm Orlicz spaces, vectorvalued Banach spaces, embeddings, interpolation, maximal operator.

2000 Mathematics Subject Classification: 46E30, 46B42, 46B70.
}

0236-5294/4/\$20.00 @ 2004 Akadémiai Kiadó, Budapest 
with equality when the space $E_{0}^{\theta} E_{1}^{1-\theta}$ has a continuous norm. The general equality

$$
\mathcal{F}\left(E_{0}\left(X_{0}\right), E_{1}\left(X_{1}\right)\right)=\mathcal{F}\left(E_{0}, E_{1}\right)\left(\mathcal{F}\left(X_{0}, X_{1}\right)\right)
$$

for some functors $\mathcal{F}$ was investigated by several authors (see [9] and the references given there). We consider here the case of mixed norm spaces and the Calderón-Lozanovskil construction as functor $\mathcal{F}$.

The first result, in the case of the power function $\varphi_{\theta}(u, v)=u^{1-\theta} v^{\theta}$, was proved by Bukhvalov in 1987 ([9], Theorem 3): if $E_{0}, E_{1}$ are Banach ideal spaces on $(T, \mu)$ and $F_{0}, F_{1}$ are Banach ideal spaces on $(S, \nu)$, then

$$
\left(E_{0}\left[F_{0}\right]\right)^{1-\theta}\left(E_{1}\left[F_{1}\right]\right)^{\theta} \stackrel{1}{\hookrightarrow} E_{0}^{1-\theta} E_{1}^{\theta}\left[F_{0}^{1-\theta} F_{1}^{\theta}\right],
$$

and equality in (1) holds if either the measure $\mu$ is discrete or $F_{0}$ and $F_{1}$ have the Fatou property or $F_{0}$ has a continuous norm. Bukhvalov also proved (see [8], p. 95) that

$$
\mathcal{F}\left(E_{0}[F], E_{1}[F]\right)=\mathcal{F}\left(E_{0}, E_{1}\right)[F]
$$

for any interpolation functor $\mathcal{F}$ and since for the real method it is not true that $\mathcal{F}\left(E\left[F_{0}\right], E\left[F_{0}\right]\right)=E\left[\mathcal{F}\left(F_{0}, F_{1}\right)\right]$ he considered such an equality for the Calderón-Lozanovskii construction. He also proved (see [9], Theorem 6) that the formula

$$
\varphi\left(E\left[F_{0}\right], E\left[F_{1}\right]\right)=E\left[\varphi\left(F_{0}, F_{1}\right)\right]
$$

is true if either the measure $\mu$ is discrete of if $F_{0}$ and $F_{1}$ have the Fatou property.

Of course, we would like to have the equality

$$
\mathcal{F}\left(E_{0}\left[F_{0}\right], E_{1}\left[F_{1}\right]\right)=\mathcal{F}\left(E_{0}, E_{1}\right)\left[\mathcal{F}\left(F_{0}, F_{1}\right)\right],
$$

but this is rarely satisfied.

The main purpose of this paper is to find necessary and sufficient conditions on $\varphi, \varphi_{0}, \varphi_{1}$ under which we have a continuous embedding

$$
\varphi\left(E_{0}\left[F_{0}\right], E_{1}\left[F_{1}\right]\right) \stackrel{C}{\hookrightarrow} \varphi_{0}\left(E_{0}, E_{1}\right)\left[\varphi_{1}\left(F_{0}, F_{1}\right)\right]
$$

and also the reverse embedding for any Banach ideal spaces $E_{0}, E_{1}$ on $(T, \mu)$ and $F_{0}, F_{1}$ on $(S, \nu)$. The necessary and sufficient condition for (4) is the inequality

$$
\varphi(1, u v) \leqq C \varphi_{0}(1, u) \varphi_{1}(1, v) \quad \text { for all } \quad u, v>0
$$


or the reverse inequality to (5) in the reverse embedding case.

In particular, we will get characterizations of the embedding of Orlicz spaces $L_{M}(S \times T) \subset L_{M_{0}}(T)\left[L_{M_{1}}(S)\right]$ and its reverse in terms of the functions $M, M_{0}$ and $M_{1}$. These results were considered in several papers and with different proofs (see [12], [17], [16], [26], [37]).

Moreover, we can see from the characterization of the embedding (4) and its reverse that the Calderón-Lozanovskil construction $\varphi(\cdot)$ commutes with the mixed norm spaces, that is, the equality

$$
\varphi\left(E_{0}\left[F_{0}\right], E_{1}\left[F_{1}\right]\right)=\varphi\left(E_{0}, E_{1}\right)\left[\varphi\left(F_{0}, F_{1}\right)\right]
$$

holds for arbitrary Banach ideal spaces $E_{0}, E_{1}$ on $(T, \mu)$ and $F_{0}, F_{1}$ on $(S, \nu)$ if and only if $\varphi$ is equivalent to the power function $\varphi_{\theta}(u, v)=u^{1-\theta} v^{\theta}$. This description for power functions gives equality in (1). Equalities in (1), (2) and (4) together with the interpolation property of the Calderón-Lozanovskiu construction on Banach ideal spaces with the Fatou property (cf. [35], [36], [31], [4]) give interpolation theorems for mixed norm spaces.

The Calderón-Lozanovskiǔ construction on weighted Banach ideal spaces was investigated in [25].

The content of the paper is as follows. In Section 1 we define the mixed norm space and the Calderón-Lozanovskiul construction on the class of Banach ideal spaces. Section 2 contains the main results of the paper. The embedding (4) and its reverse are characterized in terms of the functions $\varphi, \varphi_{0}$ and $\varphi_{1}$. The results obtained here are then applied to prove that the equality (6) holds if and only if $\varphi$ is equivalent to the power function. In Section 3 these results are used for the mixed norm Orlicz spaces and we obtain here, as particular cases, known theorems proved before by several authors. Finally, in Section 4 we are showing how the interpolation property of the Calderón-Lozanovskil construction on spaces with the Fatou property can be used in possible applications to classical operators between mixed norm spaces. More precisely, we can obtain results on boundedness between mixed norm Orlicz spaces from the boundedness on mixed norm $L_{p}\left[L_{q}\right]$-estimates. As a concrete example, the maximal operators are presented.

\section{Mixed norm spaces and the Calderón-Lozanovskiî construction}

We recall some notions and definitions which we will need further. Let $(T, \Sigma, \mu)$ be a complete $\sigma$-finite measure space, and let $L^{0}(\mu)$ denote, as usual, the space of all equivalence classes of measurable functions on $\Omega$ with the topology of convergence in measure on $\mu$-finite sets. The order $|x| \leqq|y|$ means that $|x(t)| \leqq|y(t)|$ for $\mu$-almost all $t \in T$. 
If a Banach subspace $E=(E,\|\cdot\|)$ of $L^{0}(\mu)$ is such that there exists $u \in E$ with $u>0 \mu$-a.e. on $T$ and $\|x\| \leqq\|y\|$ whenever $|x| \leqq|y|$, we say that $E$ is a Banach ideal space (on $T$ or on $(T, \mu)$ ).

A Banach ideal space $E$ has the Fatou property if $0 \leqq x_{n} \uparrow x, x_{n} \in E$ and $\sup _{n}\left\|x_{n}\right\|_{E}<\infty$ implies $x \in E$ and $\left\|x_{n}\right\|_{E} \uparrow\|x\|_{E}$. A Banach ideal space is said to have continuous norm if $\left\|x_{n}\right\|_{E} \downarrow 0$ whenever $0 \leqq x_{n} \in E$ and $x_{n} \downarrow 0$.

The Köthe dual or the associate space $E^{\prime}$ to a Banach ideal space $E$ is the space of all $x \in L^{0}(\mu)$ such that $\int_{T}|x(t) y(t)| d \mu<\infty$ for every $y \in Y$ endowed with the norm

$$
\|x\|_{E^{\prime}}=\sup \left\{\int_{T}|x(t) y(t)| d \mu:\|y\|_{E} \leqq 1\right\} .
$$

$E^{\prime}$ is a Banach ideal space. We have the embedding $E \subset E^{\prime \prime}$ with $\|x\|_{E^{\prime \prime}}$ $\leqq\|x\|_{E}$ for every $x \in E$. Moreover, $E=E^{\prime \prime}$ with equality of the norms if and only if $E$ has the Fatou property (cf. [24], [27]).

If $0<u \in E$, then the principal ideal $E(u)$ in $E$ is

$$
E(u)=\{x \in E: \exists \lambda>0 \text { with }|x(t)| \leqq \lambda u(t) \text { for } t \in T \mu \text {-a.e. }\}
$$

with the norm

$$
\||| x \mid\|=\inf \{\lambda>0:|x(t)| \leqq \lambda u(t) \text { for } \mu \text {-a.e. } t \in T\} .
$$

The space $E(u)$ is a Banach ideal space which is an AM-space and $\|x \mid\|$ $\geqq\|x\|$ for $x \in E(u)$ with $\|u\|\|=\| u \|$. Moreover, the space $(E(u),\|\| \cdot \|)$ has the Fatou property since the unit ball in $E(u)$ is an interval which is closed and by $[20$, Lemma 5, p. 143] we obtain that $E(u)$ has the Fatou property.

Let $E$ be a Banach ideal space on $(T, \mu)$ and let $X$ be a Banach space. We denote by $E(X)$ the set of all strongly-measurable functions $x=x(\cdot)$ : $T \rightarrow X$ such that $\|x(\cdot)\|_{X} \in E$. This is a Banach space under pointwise operations and a natural norm given by

$$
\|x\|_{E(X)}:=\|\| x(\cdot)\left\|_{X}\right\|_{E} .
$$

The space $E(X)$ is strictly connected with the mixed norm space.

Consider two Banach ideal spaces $E$ and $F$ on $(T, \Sigma, \mu)$ and $(S, \Lambda, \nu)$, respectively, and assume all the time (for measurability reasons) that either the measure $\nu$ is discrete or the norm $\|\cdot\|_{F}$ is semi-continuous, i.e., if $0 \leqq x_{n} \uparrow x \in E$, then $\left\|x_{n}\right\|_{E} \uparrow\|x\|_{E}$. 
Define a mixed norm Banach ideal space $E[F]$ on $S \times T$ as classes of all $\nu \times \mu$ measurable functions $K=\{K(s, t), s \in S, t \in T\}$ on $S \times T$ such that $K(\cdot, t) \in F$ for $\mu$-almost all $t \in T$ and $\|K(\cdot, t)\|_{F} \in E$ with the norm

$$
\|K\|_{E[F]}:=\|\| K(s, t)\left\|_{F, s}\right\|_{E, t} \text {. }
$$

How are the spaces $E(F)$ and $E[F]$ connected? Any vector-valued function $x(\cdot): T \rightarrow F$ can be considered as a function of two variables $[x(t)](s)=$ $x(s, t)$ for almost all $t$ and $s$. Always we have the embedding $E(F) \subset E[F]$ and equality holds if and only if either the measure $\mu$ on $T$ is discrete or $F$ has a continuous norm (cf. Bukhvalov [5]).

The spaces $L_{p}\left[L_{q}\right]$ are sometimes called spaces of Hille-Tamarkin kernels. In particular, we have that $L_{p}\left(L_{q}\right)=L_{p}\left[L_{q}\right]$ for $1 \leqq p, q<\infty$ and $L_{p}\left(L_{\infty}\right)$ $\neq L_{p}\left[L_{\infty}\right]$

The Köthe duality $(E[F])^{\prime}=E^{\prime}\left[F^{\prime}\right]$ with equality of the norms was proved by Blozinski ([3], Theorem 3.12) and Bukhvalov ([6], Theorem 3.1).

Now we give the definition of the Calderón-Lozanovskir spaces sometimes also called the Calderón-Lozanovskiu construction. Let $\bar{E}=\left(E_{0}, E_{1}\right)$ be a pair of Banach ideal spaces on $(T, \mu)$ and let $\mathcal{U}$ denote the set of all positive concave and positively homogeneous continuous functions $\varphi:[0, \infty) \times[0, \infty)$ $\rightarrow[0, \infty)$ such that $\varphi(0,0)=0$. Then the Calderón-Lozanovski乞 ideal space or the Calderón-Lozanovskiu construction $\varphi(\bar{E})=\varphi\left(E_{0}, E_{1}\right)$ consists of all $x \in L^{0}(\mu)$ such that $|x| \leqq \lambda \varphi\left(\left|x_{0}\right|,\left|x_{1}\right|\right) \mu$-a.e. for some $x_{i} \in E_{i}$, with $\left\|x_{i}\right\|_{E_{i}}$ $\leqq 1, i=0,1$. The space $\varphi(\bar{E})$ is a Banach ideal space equipped with the norm

$$
\|x\|_{\varphi}=\inf \left\{\lambda>0:|x| \leqq \lambda \varphi\left(\left|x_{0}\right|,\left|x_{1}\right|\right),\left\|x_{0}\right\|_{E_{0}} \leqq 1,\left\|x_{1}\right\|_{E_{1}} \leqq 1\right\}
$$

(see [28]). In the case of the power function $\varphi_{\theta}(s, t)=s^{1-\theta} t^{\theta}$ with $0 \leqq \theta \leqq 1$, $\varphi_{\theta}(\bar{E})$ is the well known Calderón space $E_{0}^{1-\theta} E_{1}^{\theta}$ (see [10]). Moreover, the particular case of $E^{\theta}\left(L^{\infty}\right)^{1-\theta}=E^{(p)}$ for $\theta=\frac{1}{p}, 1<p<\infty$ is known as the $p$-convexification of $E$ (see [27]).

The properties of $\varphi(\bar{E})$ were studied by Lozanovskiu in [28] and [29] (see also [31] and [4]), where among other facts it was proved that the Köthe duality result $\varphi\left(E_{0}, E_{1}\right)^{\prime}=\hat{\varphi}\left(E_{0}^{\prime}, E_{1}^{\prime}\right)$ holds with equivalent norms. Here, for $\varphi \in \mathcal{U}$, the conjugate function $\hat{\varphi}$ is defined by

$$
\hat{\varphi}(u, v):=\inf \left\{\frac{\alpha u+\beta v}{\varphi(\alpha, \beta)} ; \alpha, \beta>0\right\}, \quad u, v \geqq 0 .
$$


We have $\hat{\varphi} \in \mathcal{U}$ and $\hat{\hat{\varphi}}=\varphi$ (see [29], [30] and [31]).

The Calderón-Lozanovskiu spaces are closely related to Orlicz spaces. Let $M:[0, \infty) \rightarrow[0, \infty]$ be an Orlicz function, that is, a nondecreasing, convex and left-continuous function not identical to 0 or $\infty$ on $(0, \infty)$ with $M(0)=0$. For the function $\varphi$ defined by $\varphi(u, v)=v M^{-1}(u / v)$ if $v>0$ and 0 if $v=0$, where $M^{-1}$ is the right continuous inverse of $M$, we have $\varphi \in \mathcal{U}$ and then for any Banach ideal space $E$, the Calderón-Lozanovskiu space $\varphi\left(E, L_{\infty}\right)$ coincides isometrically with the Banach ideal space

$$
E_{M}=\left\{x \in L^{0}(\mu) ; M(|x| / \lambda) \in E \text { for some } \lambda>0\right\}
$$

equipped with the norm

$$
\|x\|_{E_{M}}=\inf \left\{\lambda>0 ;\|M(|x| / \lambda)\|_{E} \leqq 1\right\} .
$$

In particular, $\varphi\left(L_{1}, L_{\infty}\right)$ coincides isometrically with the Orlicz space $L_{M}$ (see [4], [31], [36]). If the Orlicz function $M$ satisfies the so-called $\Delta_{2}$-condition, i.e., $M(2 u) \leqq C M(u)$ for some $C \geqq 1$ and all $u \in(0, \infty)$, then the Orlicz space $L_{M}$ has a continuous norm.

The subset of functions in $\mathcal{U}$ for which $\varphi(u, 1) \rightarrow 0$ and $\varphi(1, v) \rightarrow 0$ as $u \rightarrow 0^{+}$and $v \rightarrow 0^{+}$is denoted by $\mathcal{U}_{0}$. The subsets of functions in $\mathcal{U}$ for which $\bar{\varphi}(u, 1) \rightarrow 0$ as $u \rightarrow 0^{+}$are denoted by $\mathcal{U}^{-}$and the other for which $\bar{\varphi}(1, v) \rightarrow 0$ as $v \rightarrow 0^{+}$by $\mathcal{U}^{+}$, where $\bar{\varphi}(u, v)=\sup _{a, b>0} \frac{\varphi(u a, v b)}{\varphi(a, b)}$.

Note that $\varphi \in \mathcal{U}^{-}$if and only if the upper Orlicz-Matuszewska index of $\varphi(\cdot):=\varphi(1, \cdot)$ is strictly less than 1 , i.e., $\beta_{\varphi}<1$ and $\varphi \in \mathcal{U}^{+}$if and only if the lower Orlicz-Matuszewska index of $\varphi$ is strictly bigger than 0, i.e., $\alpha_{\varphi}>0$ (cf. [24], [31]).

It is true that if either $E_{0}$ has a continuous norm and $\beta_{\varphi}<1$ or $E_{1}$ has a continuous norm and $\alpha_{\varphi}>0$, then $\varphi\left(E_{0}, E_{1}\right)$ has a continuous norm (see [31], Theorem 15.10).

\section{The Calderón-Lozanovskiǔ construction for mixed norm spaces}

The first result, connected with equality (2), was mentioned in [9] but we present here the proof for the sake of completeness.

Lemma 1. Let $\varphi \in \mathcal{U}$. Then

$$
\varphi\left(E_{0}[F], E_{1}[F]\right)=\varphi\left(E_{0}, E_{1}\right)[F]
$$


with equality of the norms for any Banach ideal spaces $E_{0}, E_{1}$ on $(T, \mu)$ and $F$ on $(S, \nu)$.

Proof. If $K \in \varphi\left(E_{0}[F], E_{1}[F]\right)$, then

$$
|K(s, t)| \leqq \lambda \varphi\left(\left|x_{0}(s, t)\right|,\left|x_{1}(s, t)\right|\right) \leqq \lambda \frac{a\left|x_{0}(s, t)\right|+b\left|x_{1}(s, t)\right|}{\hat{\varphi}(a, b)}
$$

for almost all $s \in S, t \in T$ and any $a, b>0$ with $\left\|x_{i}\right\|_{E_{i}[F]} \leqq 1, i=0,1$. Therefore

$$
\|K(s, t)\|_{F} \leqq \lambda \frac{a\left\|x_{0}(s, t)\right\|_{F}+b\left\|x_{1}(s, t)\right\|_{F}}{\hat{\varphi}(a, b)}
$$

for almost all $t \in T$ and any $a, b>0$. Since $\hat{\hat{\varphi}}=\varphi$ it follows that

$$
\|K(s, t)\|_{F} \leqq \lambda \varphi\left(\left\|x_{0}(s, t)\right\|_{F},\left\|x_{1}(s, t)\right\|_{F}\right)=\lambda \varphi\left(u_{0}(t), u_{1}(t)\right)
$$

with $u_{i}(t):=\left\|x_{i}(\cdot, t)\right\|_{F} \in E_{i}$ and $\left\|u_{i}\right\|_{E_{i}} \leqq 1, i=0,1$. This means that $\|K(\cdot, t)\|_{F} \in \varphi\left(E_{0}, E_{1}\right)$ and

$$
\|K(s, t)\|_{\varphi\left(E_{0}, E_{1}\right)[F]}=\|\| K(\cdot, t)\left\|_{F}\right\|_{\varphi\left(E_{0}, E_{1}\right)} \leqq \lambda,
$$

which gives the embedding

$$
\varphi\left(E_{0}[F], E_{1}[F]\right) \stackrel{1}{\hookrightarrow} \varphi\left(E_{0}, E_{1}\right)[F] .
$$

On the other hand, if $K \in \varphi\left(E_{0}, E_{1}\right)[F]$ then $K(\cdot, t) \in F,\|K(\cdot, t)\|_{F} \in$ $\varphi\left(E_{0}, E_{1}\right)$ and so

$$
\|K(\cdot, t)\|_{F} \leqq \lambda \varphi\left(\left|x_{0}(t)\right|,\left|x_{1}(t)\right|\right) \quad \text { with } \quad\left\|x_{i}\right\|_{E_{i}} \leqq 1, \quad i=0,1 .
$$

Let $A=\left\{t \in T:\|K(\cdot, t)\|_{F}>0\right\}$ and

$$
K_{i}(s, t)=\frac{x_{i}(t)}{\|K(\cdot, t)\|_{F}} \chi_{A}(t) K(s, t), \quad i=0,1 .
$$

Then

$$
|K(s, t)| \leqq \frac{\lambda \varphi\left(\left|x_{0}(t)\right|,\left|x_{1}(t)\right|\right)}{\|K(\cdot, t)\|_{F}} \chi_{A}(t) K(s, t)=\lambda \varphi\left(\left|K_{0}(s, t)\right|,\left|K_{1}(s, t)\right|\right)
$$


for almost all $s \in S, t \in T$ and $\left\|K_{i}\right\|_{E_{i}[F]}=\|\| K_{i}(\cdot, t)\left\|_{F}\right\|_{E_{i}} \leqq\left\|x_{i}\right\|_{E_{i}} \leqq 1$. Therefore, $K \in \varphi\left(E_{0}[F], E_{1}[F]\right)$ and $\|K\| \leqq \lambda$. From this it follows that the embedding

$$
\varphi\left(E_{0}, E_{1}\right)[F] \stackrel{1}{\hookrightarrow} \varphi\left(E_{0}[F], E_{1}[F]\right)
$$

holds. The proof is complete.

Immediately from Lemma 1 , by taking $\varphi(s, t)=\min (s, t)$ and $\varphi(s, t)$ $=\max (s, t)$ we obtain the following result (cf. [15], Proposition 4.4 for the sum):

Corollary 1. We have $E_{0}[F] \cap E_{1}[F]=\left(E_{0} \cap E_{1}\right)[F]$ with equality of the norms and $E_{0}[F]+E_{1}[F]=\left(E_{0}+E_{1}\right)[F]$ with equivalent norms.

We consider now the continuous inclusions for the Calderón-Lozanovskiı construction of mixed norm spaces.

Theorem 1. Let $\varphi, \varphi_{0}, \varphi_{1} \in \mathcal{U}$. The embedding

$$
\varphi\left(E_{0}\left[F_{0}\right], E_{1}\left[F_{1}\right]\right) \stackrel{C}{\hookrightarrow} \varphi_{0}\left(E_{0}, E_{1}\right)\left[\varphi_{1}\left(F_{0}, F_{1}\right)\right]
$$

holds for any Banach ideal spaces $E_{0}, E_{1}$ on $(T, \mu)$ and $F_{0}, F_{1}$ on $(S, \nu)$ if and only if

$$
\varphi(1, u v) \leqq C \varphi_{0}(1, u) \varphi_{1}(1, v) \quad \text { for all } \quad u, v>0 .
$$

Proof. Sufficiency. Assume that (8) is true. Suppose that $K \in$ $\varphi\left(E_{0}\left[F_{0}\right], E_{1}\left[F_{1}\right]\right)$ and has norm $\|K\|$. Then for any $\varepsilon>0$ there are $K_{i}$ $\in E_{i}\left[F_{i}\right]$ with $\left\|K_{i}\right\|_{E_{i}\left[F_{i}\right]} \leqq 1, i=0,1$, such that

$$
|K(s, t)| \leqq(\|K\|+\varepsilon) \varphi\left(\left|K_{0}(s, t)\right|,\left|K_{1}(s, t)\right|\right) .
$$

Hence, by (8),

$$
\begin{aligned}
& \frac{|K(s, t)|}{\|K\|+\varepsilon}=\varphi\left(\left\|K_{0}(\cdot, t)\right\|_{F_{0}} \frac{\left|K_{0}(s, t)\right|}{\left\|K_{0}(\cdot, t)\right\|_{F_{0}}},\left\|K_{1}(\cdot, t)\right\|_{F_{1}} \frac{\left|K_{1}(s, t)\right|}{\left\|K_{1}(\cdot, t)\right\|_{F_{1}}}\right) \\
& \leqq C \varphi_{0}\left(\left\|K_{0}(\cdot, t)\right\|_{F_{0}},\left\|K_{1}(\cdot, t)\right\|_{F_{1}}\right) \varphi_{1}\left(\frac{\left|K_{0}(s, t)\right|}{\left\|K_{0}(\cdot, t)\right\|_{F_{0}}}, \frac{\left|K_{1}(s, t)\right|}{\left\|K_{1}(\cdot, t)\right\|_{F_{1}}}\right) .
\end{aligned}
$$

Since, for $\mu$-almost all $t \in T$,

$$
u_{t}(s):=\varphi_{1}\left(\frac{\left|K_{0}(s, t)\right|}{\left\|K_{0}(\cdot, t)\right\|_{F_{0}}}, \frac{\left|K_{1}(s, t)\right|}{\left\|K_{1}(\cdot, t)\right\|_{F_{1}}}\right)=\varphi_{1}\left(u_{t}^{0}(s), u_{t}^{1}(s)\right)
$$


and $\left\|u_{t}^{i}\right\|_{F_{i}}=1$ for $i=0,1$ it follows that $u_{t} \in \varphi_{1}\left(F_{0}, F_{1}\right)$ and $\left\|u_{t}\right\|_{\varphi_{1}} \leqq 1$. Thus

$$
|K(s, t)| \leqq C(\|K\|+\varepsilon) \varphi_{0}\left(\left\|K_{0}(\cdot, t)\right\|_{F_{0}},\left\|K_{1}(\cdot, t)\right\|_{F_{1}}\right) \times u_{t}(s)
$$

and

$$
\begin{gathered}
v(t):=\|K(\cdot, t)\|_{\varphi_{1}\left(F_{0}, F_{1}\right)} \\
\leqq C(\|K\|+\varepsilon) \varphi_{0}\left(\left\|K_{0}(\cdot, t)\right\|_{F_{0}},\left\|K_{1}(\cdot, t)\right\|_{F_{1}}\right)\left\|u_{t}\right\|_{\varphi_{1}\left(F_{0}, F_{1}\right)} \\
\leqq C(\|K\|+\varepsilon) \varphi_{0}\left(\left\|K_{0}(\cdot, t)\right\|_{F_{0}},\left\|K_{1}(\cdot, t)\right\|_{F_{1}}\right) \\
=C(\|K\|+\varepsilon) \varphi_{0}\left(v_{0}(t), v_{1}(t)\right)
\end{gathered}
$$

where $v_{i}(t)=\left\|K_{i}(\cdot, t)\right\|_{F_{i}}$ with $\left\|v_{i}\right\|_{E_{i}} \leqq 1$ for $i=0,1$. Therefore $v \in$ $\varphi_{0}\left(E_{0}, E_{1}\right)$ with $\|v\|_{\varphi_{0}\left(E_{0}, E_{1}\right)} \leqq C(\|K\|+\varepsilon)$ or

$$
\|\| K\left\|_{\varphi_{1}\left(F_{0}, F_{1}\right)}\right\|_{\varphi_{0}\left(E_{0}, E_{1}\right)} \leqq C(\|K\|+\varepsilon) .
$$

Since $\varepsilon>0$ was arbitrary we obtain

$$
\|K\|_{\varphi_{0}\left(E_{0}, E_{1}\right)\left[\varphi_{1}\left(F_{0}, F_{1}\right)\right]} \leqq C\|K\| .
$$

Necessity. We consider special Banach ideal spaces $E_{0}=F_{0}=L_{\infty}(I)$, $E_{1}=F_{1}=L_{1}(I)$ with $I=(0, \infty)$ and special functions

$$
K(s, t)=\chi_{A}(s) \chi_{B}(t),
$$

where $A, B$ are any subsets of $I$ of positive finite measure. Then

$$
\varphi_{i}\left(L_{\infty}, L_{1}\right)=L_{M_{i}}(I), \quad \varphi\left(L_{\infty}\left[L_{\infty}\right], L_{1}\left[L_{1}\right]\right)=L_{M}(I \times I),
$$

with $\varphi_{i}(s, t)=s M_{i}^{-1}(t / s), i=0,1$, and $\varphi(s, t)=s M^{-1}(t / s)$ for $s>0$. Moreover,

$$
\begin{gathered}
\|K(\cdot, t)\|_{\varphi_{1}\left(L_{\infty}, L_{1}\right)}=\|K(\cdot, t)\|_{L_{M_{1}}}=\left\|\chi_{A}\right\|_{L_{M_{1}}} \chi_{B}(t) \\
=\frac{1}{M_{1}^{-1}(1 / m A)} \chi_{B}(t)=\frac{1}{\varphi_{1}\left(1, \frac{1}{m A}\right)} \chi_{B}(t)
\end{gathered}
$$


and

$$
\|\| K(\cdot, t)\left\|_{\varphi_{1}}\right\|_{\varphi_{0}}=\frac{1}{\varphi_{1}\left(1, \frac{1}{m A}\right) \varphi_{0}\left(1, \frac{1}{m B}\right)}
$$

Also

$$
\begin{gathered}
\|K(s, t)\|_{\varphi\left(L_{\infty}\left[L_{\infty}\right], L_{1}\left[L_{1}\right]\right)}=\left\|\chi_{A}(s) \chi_{B}(t)\right\|_{L_{M}(I \times I)} \\
=\inf \left\{\lambda>0: \int_{0}^{\infty} \int_{0}^{\infty} M\left(\chi_{A}(s) \chi_{B}(t) / \lambda\right) \leqq 1\right\} \\
=\frac{1}{M^{-1}\left(\frac{1}{m A} \frac{1}{m B}\right)}=\frac{1}{\varphi\left(1, \frac{1}{m A m B}\right)} .
\end{gathered}
$$

Thus from (7) we obtain

$$
\varphi\left(1, \frac{1}{m A m B}\right) \leqq C \varphi_{1}\left(1, \frac{1}{m A}\right) \varphi_{0}\left(1, \frac{1}{m B}\right) .
$$

Since $A$ and $B$ were arbitrary subsets of $I=(0, \infty)$ we can take $u=\frac{1}{m B}$, $v=\frac{1}{m A}$ and then the last estimate is exactly the estimate (8), which means that also the proof of the necessity is finished.

Corollary 2. If $\varphi \in \mathcal{U}$ is a C-submultiplicative function, then the embedding

$$
\varphi\left(E_{0}\left[F_{0}\right], E_{1}\left[F_{1}\right]\right) \stackrel{C}{\hookrightarrow} \varphi\left(E_{0}, E_{1}\right)\left[\varphi\left(F_{0}, F_{1}\right)\right]
$$

holds for any Banach ideal spaces $E_{0}, E_{1}$ on $(T, \mu)$ and $F_{0}, F_{1}$ on $(S, \nu)$. In particular, we always have the embedding

$$
\left(E_{0}\left[F_{0}\right]\right)^{1-\theta}\left(E_{1}\left[F_{1}\right]\right)^{\theta} \stackrel{1}{\hookrightarrow} E_{0}^{1-\theta} E_{1}^{\theta}\left[F_{0}^{1-\theta} F_{1}^{\theta}\right] .
$$

REMARK 1. In Theorem 1 the assumption (8) gives by its symmetry the embedding

$$
\varphi\left(E_{0}\left[F_{0}\right], E_{1}\left[F_{1}\right]\right) \stackrel{C}{\hookrightarrow} \varphi_{0}\left(E_{0}, E_{1}\right)\left[\varphi_{1}\left(F_{0}, F_{1}\right)\right] \cap \varphi_{1}\left(E_{0}, E_{1}\right)\left[\varphi_{0}\left(F_{0}, F_{1}\right)\right] .
$$

This result is also true for quasi-Banach ideal spaces $E_{0}, E_{1}$ on $(T, \mu)$ and $F_{0}, F_{1}$ on $(S, \nu)$; we only should mention here that the Calderón-Lozanovskiu spaces can be defined also in this case (cf. [33], [19]). 
REMARK 2. If we know something more about the spaces $F_{0}, F_{1}$, then the proof of Theorem 1 gives also the following result: Let one of the following two conditions be satisfied:

(i) $F_{0} \hookrightarrow F_{1}$ with $\|x\|_{F_{1}} \leqq a\|x\|_{F_{0}}$ for all $x \in F_{0}$ and

$$
\varphi(1, u v) \leqq C \varphi_{0}(1, u) \varphi_{1}(1, v)
$$

for a certain $C>0$ and all $0<u \leqq a, v>0$,

(ii) $F_{1} \hookrightarrow F_{0}$ with $\|x\|_{F_{0}} \leqq b\|x\|_{F_{1}}$ for all $x \in F_{1}$ and $(*)$ holds for a certain $C>0$ and all $u \geqq \frac{1}{b}, v>0$.

Then the embedding

$$
\varphi\left(E_{0}\left[F_{0}\right], E_{1}\left[F_{1}\right]\right) \stackrel{C}{\hookrightarrow} \varphi_{0}\left(E_{0}, E_{1}\right)\left[\varphi_{1}\left(F_{0}, F_{1}\right)\right]
$$

holds for any Banach ideal spaces $E_{0}, E_{1}$.

Bukhvalov ([5], Theorem 2 which is without proof; [9], Theorem 6) proved that the formula

$$
\varphi\left(E\left[F_{0}\right], E\left[F_{1}\right]\right)=E\left[\varphi\left(F_{0}, F_{1}\right)\right]
$$

holds if either the measure $\mu$ is discrete or $F_{0}$ and $F_{1}$ have the Fatou property or $F_{0}$ has a continuous norm and $\beta_{\varphi}<1$. In particular, we have the equalities $E\left[F_{0}\right] \cap E\left[F_{1}\right]=E\left[F_{0} \cap F_{1}\right]$ and $E\left[F_{0}\right]+E\left[F_{1}\right]=E\left[F_{0}+F_{1}\right]$ with equivalent norms (cf. [15], Proposition 4.6 for the sum). The following generalization is true:

Theorem 2. Let $\varphi, \varphi_{0}, \varphi_{1} \in \mathcal{U}$. Assume that one of the following four conditions is satisfied:

(i) the measure $\mu$ is discrete,

(ii) $F_{0}$ and $F_{1}$ have the Fatou property,

(iii) $F_{0}$ has a continuous norm and $\beta_{\varphi_{1}}<1$.

(iv) $F_{1}$ has a continuous norm and $\alpha_{\varphi_{1}}>0$.

Then the embedding

$$
\varphi_{0}\left(E_{0}, E_{1}\right)\left[\varphi_{1}\left(F_{0}, F_{1}\right)\right] \stackrel{C}{\hookrightarrow} \varphi\left(E_{0}\left[F_{0}\right], E_{1}\left[F_{1}\right]\right)
$$

holds for any Banach ideal spaces $E_{0}, E_{1}$ on $(T, \mu)$ and $F_{0}, F_{1}$ on $(S, \nu)$ if and only if

$$
\varphi_{0}(1, u) \varphi_{1}(1, v) \leqq C \varphi(1, u v) \quad \text { for all } \quad u, v>0
$$


Proof. Necessity. We can get (11) by the same considerations as in the proof of Theorem 1.

Sufficiency. First case. Assume that (11) holds and assume additionally that $E_{0}, E_{1}, F_{0}$ and $F_{1}$ have the Fatou property. Then

$$
\hat{\varphi}(1, u v) \leqq C \hat{\varphi}_{0}(1, u) \hat{\varphi}_{1}(1, v) \text { for all } \quad u, v>0 .
$$

In fact, by (11), we have

$$
\begin{gathered}
\hat{\varphi}_{0}(1, u) \hat{\varphi}_{1}(1, v)=\inf _{\alpha, \beta>0} \frac{\alpha+\beta u}{\varphi_{0}(\alpha, \beta)} \cdot \inf _{\gamma, \delta>0} \frac{\gamma+\delta v}{\varphi_{1}(\gamma, \delta)} \\
\geqq \frac{1}{C} \inf _{\alpha, \beta, \gamma, \delta>0} \frac{\alpha \gamma+\beta \gamma u+\alpha \delta v+\beta \delta u v}{\varphi(\alpha \gamma, \beta \delta)} \geqq \frac{1}{C} \hat{\varphi}(1, u v) .
\end{gathered}
$$

By using Theorem 1 we obtain

$$
\hat{\varphi}\left(E_{0}^{\prime}\left[F_{0}^{\prime}\right], E_{1}^{\prime}\left[F_{1}^{\prime}\right]\right) \stackrel{C}{\hookrightarrow} \hat{\varphi}_{0}\left(E_{0}^{\prime}, E_{1}^{\prime}\right)\left[\hat{\varphi}_{1}\left(F_{0}^{\prime}, F_{1}^{\prime}\right)\right]
$$

and by taking the Köthe dual

$$
G=\left(\hat{\varphi}_{0}\left(E_{0}^{\prime}, E_{1}^{\prime}\right)\left[\hat{\varphi}_{1}\left(F_{0}^{\prime}, F_{1}^{\prime}\right)\right]\right)^{\prime} \stackrel{C}{\hookrightarrow}\left(\hat{\varphi}\left(E_{0}^{\prime}\left[F_{0}^{\prime}\right], E_{1}^{\prime}\left[F_{1}^{\prime}\right]\right)\right)^{\prime}=H .
$$

Now, using the Blozinski-Bukhvalov and Lozanovskiı̌ duality theorems we obtain

$$
\begin{gathered}
G=\hat{\varphi}_{0}\left(E_{0}^{\prime}, E_{1}^{\prime}\right)^{\prime}\left[\hat{\varphi}_{1}\left(F_{0}^{\prime}, F_{1}^{\prime}\right)^{\prime}\right]=\varphi_{0}\left(E_{0}^{\prime \prime}, E_{1}^{\prime \prime}\right)\left[\varphi_{1}\left(F_{0}^{\prime \prime}, F_{1}^{\prime \prime}\right)\right] \\
=\varphi_{0}\left(E_{0}, E_{1}\right)\left[\varphi_{1}\left(F_{0}, F_{1}\right)\right]
\end{gathered}
$$

and

$$
H=\varphi\left(\left(E_{0}^{\prime}\left[F_{0}^{\prime}\right]\right)^{\prime},\left(E_{1}^{\prime}\left[F_{1}^{\prime}\right]\right)^{\prime}\right)=\varphi\left(E_{0}^{\prime \prime}\left[F_{0}^{\prime \prime}\right], E_{1}^{\prime \prime}\left[F_{1}^{\prime \prime}\right]\right)=\varphi\left(E_{0}\left[F_{0}\right], E_{1}\left[F_{1}\right]\right),
$$

which gives (10).

Second case. Assume that (11) holds but we do not have additional assumptions of the Fatou property about the spaces. Suppose that $K \in$ $\varphi_{0}\left(E_{0}, E_{1}\right)\left[\varphi_{1}\left(F_{0}, F_{1}\right)\right]$ with the norm $\|K\|$. Since for $\mu$-almost all $t \in T$ we have $K(\cdot, t) \in \varphi_{1}\left(F_{0}, F_{1}\right)$ it follows that

(12) $|K(s, t)|=(1+\varepsilon)\|K(\cdot, t)\|_{\varphi_{1}\left(F_{0}, F_{1}\right)} \varphi_{1}\left(\left|x_{0}(s, t)\right|,\left|x_{1}(s, t)\right|\right), \quad \varepsilon>0$,

where $\left\|x_{i}(\cdot, t)\right\|_{F_{i}} \leqq 1, i=0,1$. 
A priori the functions $x_{i}(s, t)$ do not have to be measurable functions of two variables, which does not make it possible to prove (10) in the general case.

Suppose that the functions $x_{i}(s, t)$ in (12) can be chosen measurable. We will prove below this possibility in cases (i), (iii) and (iv).

Since $K \in \varphi_{0}\left(E_{0}, E_{1}\right)\left[\varphi_{1}\left(F_{0}, F_{1}\right)\right]$ it follows that

$$
\|K(\cdot, t)\|_{\varphi_{1}\left(F_{0}, F_{1}\right)}=(1+\varepsilon)\|K\| \varphi_{0}\left(\left|y_{0}\right|,\left|y_{1}\right|\right),
$$

where $\left\|y_{i}\right\|_{E_{i}} \leqq 1$ for $i=0,1$.

Now under the measurability assumption on $x_{i}(s, t)$ we can put

$$
K_{i}(s, t)=(1+\varepsilon)^{2}\|K\| y_{i}(t) x_{i}(s, t), \quad i=0,1 .
$$

Then, by the assumption (11), we have

$$
\begin{gathered}
|K(s, t)|=(1+\varepsilon)\|K(\cdot, t)\| \varphi_{\varphi_{1}\left(F_{0}, F_{1}\right)} \varphi_{1}\left(\left|x_{0}(s, t)\right|,\left|x_{1}(s, t)\right|\right) \\
=(1+\varepsilon)^{2}\|K\| \varphi_{0}\left(\left|y_{0}(t)\right|,\left|y_{1}(t)\right|\right) \varphi_{1}\left(\left|x_{0}(s, t)\right|,\left|x_{1}(s, t)\right|\right) \\
\leqq(1+\varepsilon)^{2}\|K\| C \varphi\left(\left|y_{0}(t) x_{0}(s, t)\right|,\left|y_{1}(t) x_{1}(s, t)\right|\right) \\
=C \varphi\left(\left|K_{0}(s, t)\right|,\left|K_{1}(s, t)\right|\right),
\end{gathered}
$$

where

$$
\left\|K_{i}(\cdot, t)\right\|_{F_{i}} \leqq(1+\varepsilon)^{2}\|K\|\left|y_{i}(t)\right|\left\|x_{i}(\cdot, t)\right\|_{F_{i}} \leqq(1+\varepsilon)^{2}\|K\|\left|y_{i}(t)\right|
$$

and so

$$
\|K\|_{E_{i}\left[F_{i}\right]} \leqq(1+\varepsilon)^{2}\|K\|,\left\|y_{i}\right\|_{E_{i}} \leqq(1+\varepsilon)^{2}\|K\| .
$$

Hence, $K \in \varphi\left(E_{0}\left[F_{0}\right], E_{1}\left[F_{1}\right]\right)$ and its norm is not bigger than $C(1+\varepsilon)^{2}\|K\|$.

We now return to the measurability assumption we have made. If $\mu$ is discrete, then the functions are clearly measurable. If either $F_{0}$ has a continuous norm and $\beta_{\varphi_{1}}<1$ or $F_{1}$ has a continuous norm and $\alpha_{\varphi_{1}}>0$, then $\varphi_{1}\left(E_{0}, E_{1}\right)$ has a continuous norm (see [31], Theorem 15.10). Thus the mixed norm space $\varphi_{0}\left(E_{0}, E_{1}\right)\left[\varphi_{1}\left(F_{0}, F_{1}\right)\right]$ is equal to the vector-valued space $\varphi_{0}\left(E_{0}, E_{1}\right)\left(\varphi_{1}\left(F_{0}, F_{1}\right)\right)$ in which countably-valued functions are dense (see [24], Lemma 4, p. 211). Therefore functions of the form

$$
K(s, t)=\sum_{n=1}^{\infty} \chi_{A_{n}}(t) y_{n}(s),
$$


where the sets $A_{n}$ are $\mu$-measurable and pairwise disjoint, $\chi_{A_{n}} \in E_{0} \cap E_{1}$, $y_{n} \in \varphi_{1}\left(F_{0}, F_{1}\right)$, are dense in $\varphi_{0}\left(E_{0}, E_{1}\right)\left[\varphi_{1}\left(F_{0}, F_{1}\right)\right]$.

Now, it is enough to show the inclusion (10) for functions of the form (14). We have

$$
\left|y_{n}\right|=(1+\varepsilon)\left\|y_{n}\right\|_{\varphi_{1}\left(F_{0}, F_{1}\right.} \varphi_{1}\left(\left|x_{0 n}\right|,\left|x_{1 n}\right|\right)
$$

with $\left\|x_{i n}\right\|_{F_{i}} \leqq 1, i=0,1$. Put

$$
x_{i}(s, t)=\sum_{n=1}^{\infty} \chi_{A_{n}}(t) x_{i n}(s)
$$

Then $x_{i}(s, t)$ are measurable and $\left\|x_{i}(\cdot, t)\right\|_{F_{i}} \leqq 1, i=0,1$. The rest of the proof is the same as above.

Suppose now that $F_{0}$ and $F_{1}$ have the Fatou property. Let $y_{0}, y_{1}$ be from (13) and consider the principal ideals $G_{i}=E_{i}\left(\left|y_{i}\right|\right), i=0,1$ which have the Fatou property. By the first case in which all the spaces have the Fatou property we have the embedding

$$
\varphi_{0}\left(G_{0}, G_{1}\right)\left[\varphi_{1}\left(F_{0}, F_{1}\right)\right] \stackrel{C}{\hookrightarrow} \varphi\left(G_{0}\left[F_{0}\right], G_{1}\left[F_{1}\right]\right) .
$$

Therefore,

$|K(s, t)| \leqq(1+\varepsilon) C\|K\| \varphi\left(K_{0}(s, t), K_{1}(s, t)\right) \quad$ with $\quad\left\|K_{i}\right\|_{G_{i}\left[F_{i}\right]} \leqq 1, i=0,1$.

Thus, for $i=0,1$, the assumption $\left\|K_{i}\right\|_{G_{i}\left[F_{i}\right]} \leqq 1$ implies $\left\|K_{i}(\cdot, t)\right\|_{F_{i}} \leqq$ $\left|y_{i}(t)\right|$ and so $\left\|K_{i}(\cdot, t)\right\|_{F_{i}} \leqq\left\|y_{i}\right\|_{E_{i}} \leqq 1$. Hence, $K \in \varphi\left(E_{0}\left[F_{0}\right], E_{1}\left[F_{1}\right]\right)$ with the required estimate of the norm. This completes the proof of Theorem 2.

Corollary 3. If $\varphi \in \mathcal{U}$ is a C-supermultiplicative function and either the measure $\mu$ is discrete or both $F_{0}$ and $F_{1}$ have the Fatou property, then the embedding

$$
\varphi\left(E_{0}, E_{1}\right)\left[\varphi\left(F_{0}, F_{1}\right)\right] \stackrel{C}{\hookrightarrow} \varphi\left(E_{0}\left[F_{0}\right], E_{1}\left[F_{1}\right]\right)
$$

holds for any Banach ideal spaces $E_{0}, E_{1}$ on $(T, \mu)$ and $F_{0}, F_{1}$ on $(S, \nu)$. In particular, we always have the embedding

$$
E_{0}^{1-\theta} E_{1}^{\theta}\left[F_{0}^{1-\theta} F_{1}^{\theta}\right] \stackrel{1}{\hookrightarrow}\left(E_{0}\left[F_{0}\right]\right)^{1-\theta}\left(E_{1}\left[F_{1}\right]\right)^{\theta} .
$$


Corollary 4. Let $\varphi \in \mathcal{U}$ and either the measure $\mu$ is discrete or $F_{0}$ and $F_{1}$ have the Fatou property. Then the equality

$$
\varphi\left(E_{0}\left[F_{0}\right], E_{1}\left[F_{1}\right]\right)=\varphi\left(E_{0}, E_{1}\right)\left[\varphi\left(F_{0}, F_{1}\right)\right]
$$

holds for any Banach ideal spaces $E_{0}, E_{1}, F_{0}, F_{1}$ if and only if $\varphi$ is equivalent to a power function.

Proof. By Theorems 1 and 2 (or Corollaries 2 and 3) the equality (15) holds if and only if there exist positive constants $c_{0}$ and $c_{1}$ such that for all $s, t>0$

$$
c_{0} \varphi(1, s) \varphi(1, t) \leqq \varphi(1, s t) \leqq c_{1} \varphi(1, s) \varphi(1, t) .
$$

Hence, $\varphi$ is equivalent to a power function (cf., for example, [16], p. 289 and [25], Theorem 3).

REMARK 3. Note that

$$
\varphi\left(L_{p_{0}}\left[L_{q_{0}}\right], L_{p_{1}}\left[L_{q_{1}}\right]\right)=L_{M}\left[L_{N}\right]
$$

where $M^{-1}(u)=\varphi\left(u^{1 / p_{0}}, u^{1 / p_{1}}\right)$ and $N^{-1}(u)=\varphi\left(u^{1 / q_{0}}, u^{1 / q_{1}}\right)$ for any $L_{p_{0}}$, $L_{p_{1}}, L_{q_{0}}, L_{q_{1}}$ spaces if and only if $\varphi$ is equivalent to a power function $\varphi_{\theta}(u, v)$ $=u^{1-\theta} v^{\theta}$ for some $0 \leqq \theta \leqq 1$.

The next result was proved by Bukhvalov ([9], Theorem 6) but now it will follow easily from Theorems 1 and 2 .

Corollary 5. Let $\varphi \in \mathcal{U}$ and let either the measure $\mu$ be discrete or let $F$ have the Fatou property. Then the equality

$$
\varphi\left(E\left[F_{0}\right], E\left[F_{1}\right]\right)=E\left[\varphi\left(F_{0}, F_{1}\right)\right]
$$

holds for any Banach ideal spaces $E, F_{0}$ and $F_{1}$.

Proof. Taking $E_{0}=E_{1}=E$ and $\varphi_{1}=\varphi, \varphi_{0}(u, v)=\max (u, v)$ we obtain that the inequality (8) holds with $C=1$ and by Theorem 1 we have the embedding $\varphi\left(E\left[F_{0}\right], E\left[F_{1}\right]\right) \subset E\left[\varphi\left(F_{0}, F_{1}\right)\right]$. Then changing in the above only the function $\varphi_{0}(u, v)=\min (u, v)$ we obtain that the inequality (11) holds with $C=1$ and by Theorem 2 we have the reverse embedding $E\left[\varphi\left(F_{0}, F_{1}\right)\right]$ $\subset \varphi\left(E\left[F_{0}\right], E\left[F_{1}\right]\right)$, which together give the equality (16).

The following corollary was announced by Milman (cf. [32], Lemma 2.1(ii)) when both of the spaces $E$ and $F$ have the Fatou property. 
COROLlary 6. Let either $E$ or $F$ have the Fatou property and $E[F] \stackrel{B}{\hookrightarrow}$ $G(S \times T)$. If for the Orlicz functions $M_{0}, M_{1}, M$ we have the estimate $M_{0}^{-1}(u) M_{1}^{-1}(v) \leqq C M^{-1}(u v)$ for all $u, v>0$, then

$$
E_{M_{0}}\left[F_{M_{1}}\right] \stackrel{B C}{\hookrightarrow} G_{M}(S \times T) .
$$

Proof. We can rewrite this result into the following form: Let either $E$ or $F$ have the Fatou property and $E[F] \stackrel{B}{\hookrightarrow} G$. If $\varphi, \varphi_{0}, \varphi_{1} \in \mathcal{U}$ satisfy $\varphi_{0}(u, 1) \varphi_{1}(v, 1) \leqq C \varphi(u v, 1)$ for all $u, v>0$, then

$$
\varphi_{0}\left(E, L_{\infty}\right)\left[\varphi_{1}\left(F, L_{\infty}\right)\right] \stackrel{B C}{\hookrightarrow} \varphi\left(G, L_{\infty}\right)
$$

and the validity of this embedding follows from Theorem 2(ii).

Cerdà and Mastyło ([11], Theorem 2.3) proved some vector-valued continuous inclusions for the Calderón-Lozanovskiu spaces in connection with the study of type and cotype. In their case the space $E$ is a Banach sequence ideal space with $E \subset l_{\infty}$. We will show how, from Theorem 1, their result follows for an arbitrary Banach ideal space $E$.

Corollary 7. Let $\varphi, \varphi_{0} \in \mathcal{U}$ and let $M_{0}, M_{1}, M$ be Orlicz functions. If $\varphi(1, u v) \leqq C \varphi_{0}(1, u) \varphi(1, v), \quad \varphi_{0}\left(M_{0}^{-1}(u), M_{1}^{-1}(u)\right)=M^{-1}(u)$ for all $u, v>0$, then

$$
\varphi\left(E_{M_{0}}\left[F_{0}\right], E_{M_{1}}\left[F_{1}\right]\right) \hookrightarrow E_{M}\left[\varphi\left(F_{0}, F_{1}\right)\right]
$$

for any Banach ideal spaces $E$ on $(T, \mu)$ and $F_{0}, F_{1}$ on $(S, \nu)$. In particular, if $\varphi \in \mathcal{U}$ is a $C$-submultiplicative function and $\varphi\left(M_{0}^{-1}(u), M_{1}^{-1}(u)\right)=M^{-1}(u)$ for all $u>0$, then $\varphi\left(L_{M_{0}}\left[F_{0}\right], L_{M_{1}}\left[F_{1}\right]\right) \hookrightarrow L_{M}\left[\varphi\left(F_{0}, F_{1}\right)\right]$.

Proof. By Theorem 1 we have

$$
\begin{gathered}
\varphi\left(E_{M_{0}}\left[F_{0}\right], E_{M_{1}}\left[F_{1}\right]\right)=\varphi\left(M_{0}^{-1}\left(E, L_{\infty}\right)\left[F_{0}\right], M_{1}^{-1}\left(E, L_{\infty}\right)\left[F_{1}\right]\right) \\
\hookrightarrow \varphi_{0}\left(M_{0}^{-1}\left(E, L_{\infty}\right), M_{1}^{-1}\left(E, L_{\infty}\right)\right)\left[\varphi\left(F_{0}, F_{1}\right)\right]
\end{gathered}
$$

and by the reiteration property (cf. [31], pp. 180-181) the last space is equal to

$$
M^{-1}\left(E, L_{\infty}\right)\left[\varphi\left(F_{0}, F_{1}\right)\right]=E_{M}\left[\varphi\left(F_{0}, F_{1}\right)\right] .
$$

Note that if $M$ satisfies the $\Delta_{2}$-condition then we get their assumption $\varphi \in \mathcal{U}\left(M, M_{0}, M_{1}\right)$ (cf. [11] for definition). We can also obtain, from our Theorem 2, their reverse result in Theorem 2.5. 


\section{Mixed norm Orlicz spaces}

The interest in mixed norm Orlicz spaces started already in the sixties and seventies (cf. [37] and [12], [17], [26]). Clearly, for the mixed norm $L_{p^{-}}$ spaces we have the imbedding $L_{p}(T)\left[L_{q}(S)\right] \subset L_{r}(S \times T)$ for any $\sigma$-finite measure spaces $(T, \mu)$ and $(S, \nu)$ if and only if $p=q=r$. Theorems 1 and 2 allow us to generalize this observation by characterization of the embeddings between Orlicz spaces with mixed norms and Orlicz spaces defined on a product of measure spaces.

Theorem 3. (i) The embedding

$$
L_{M_{0}}(T)\left[L_{M_{1}}(S)\right] \stackrel{C}{\hookrightarrow} L_{M}(S \times T)
$$

holds for any $\sigma$-finite measure spaces $(T, \mu)$ and $(S, \nu)$ if and only if

$$
M(C u v) \leqq M_{0}(u) M_{1}(v) \text { for all } u, v>0 .
$$

Moreover, if additionally $\mu(T)<\infty$ and $\nu(S)<\infty$, then the above embedding holds if and only if the estimate on the functions holds for all $u, v \geqq u_{0}>0$.

(ii) The embedding

$$
L_{M}(S \times T) \stackrel{C}{\hookrightarrow} L_{M_{0}}(T)\left[L_{M_{1}}(S)\right]
$$

holds for any $\sigma$-finite measure spaces $(T, \mu)$ and $(S, \nu)$ if and only if

$$
M(C u v) \geqq M_{0}(u) M_{1}(v) \text { for all } u, v>0 .
$$

Moreover, if additionally $\mu(T)<\infty$ and $\nu(S)<\infty$, then the above embedding holds if and only if the estimate on the functions holds for all $u, v \geqq u_{0}>0$.

Proof. The results follow directly from Theorems 1 and 2 by considering $E_{0}=L_{1}(\mu), E_{1}=L_{\infty}(\mu), F_{0}=L_{1}(\nu)$ and $F_{1}=L_{\infty}(\nu)$ in these theorems.

Theorem 3(i), in the case of non-atomic finite measures, was proved independently by Donaldson [12], Firley-Matuszewska [17] and Lacroix [26]. The case of non-atomic infinite measures was done by Lacroix [26], and then also by Milman [32].

Theorem 3 (ii), in the case of non-atomic finite measures, was proved by Rutickii [37] and Firley-Matuszewska [17].

COROLlary 8. The equality

$$
L_{M_{0}}(T)\left[L_{M_{1}}(S)\right]=L_{M}(S \times T)
$$


holds for any $\sigma$-finite measure spaces $(T, \mu)$ and $(S, \nu)$ if and only if there exists $p \in[1, \infty]$ such that all the Orlicz spaces are $L_{p}$-spaces for a certain $p \geqq 1$.

Proof. We can see that the above mentioned equality gives, by Theorem 3 , the equivalences $M_{0}(u) \approx M_{1}(u) \approx M(u)$ for all $u>0$ and both c-supermultiplicativity and $C$-submultiplicativity of $M$. Therefore, $M_{0}(u)$ $\approx M_{1}(u) \approx M(u) \approx u^{p}$ for a certain $p \geqq 1$, and all the spaces are $L_{p}$-spaces.

Observe that if $\mu(T)<\infty$ and $\nu(S)<\infty$ in Corollary 8 , then the equality of the spaces holds if and only if the equivalences of the functions holds for all $u, v \geqq u_{0}>0$.

Note also that the first part of Corollary 8, i.e., the equivalences $M_{0}(u)$ $\approx M_{1}(u) \approx M(u)$ were already showed in [17], Theorem 3.3, and it was fully proved in [16], Theorem 2. In [12], Proposition 1.3 it is proved that $L_{M}(T)\left[L_{M}(S)\right]=L_{M}(S \times T)$ if and only if function $M$ is equivalent to a power function $u^{p}$ for a certain $p \geqq 1$.

Taking concrete functions in Theorem 3 we obtain particular imbeddings.

COROLlary 9. The embeddings

$$
\left(L_{1} \cap L_{\infty}\right)\left[L_{M}\right] \stackrel{1}{\hookrightarrow} L_{M}(S \times T) \stackrel{2}{\hookrightarrow}\left(L_{1}+L_{\infty}\right)\left[L_{M}\right]
$$

and

$$
L_{M}\left[L_{1} \cap L_{\infty}\right] \stackrel{1}{\hookrightarrow} L_{M}(S \times T) \stackrel{2}{\hookrightarrow} L_{M}\left[L_{1}+L_{\infty}\right]
$$

hold. Moreover, if $\nu(S)<\infty$ then

$$
L_{M}\left[L_{\infty}\right] \stackrel{C}{\hookrightarrow} L_{M}(S \times T) \stackrel{2 C}{\hookrightarrow} L_{M}\left[L_{1}\right] \quad \text { with } \quad C=\max \{1, \nu(S)\}
$$

and if $\mu(T)<\infty$ then

$$
L_{\infty}\left[L_{M}\right] \stackrel{C}{\hookrightarrow} L_{M}(S \times T) \stackrel{2 C}{\hookrightarrow} L_{1}\left[L_{M}\right] \quad \text { with } \quad C=\max \{1, \mu(T)\} .
$$

Proof. The first four embeddings follow immediately from Theorem 3. The constant 2 appeared here since we understand that the space $L_{1}+L_{\infty}$ is with its natural norm $\|\cdot\|_{L_{1}+L_{\infty}}^{0}$ and the well-known equivalence in any Orlicz space $L_{M}$ (cf. [23], p. 80 or [31], pp. 98-99)

$$
\|x\|_{L_{M}} \leqq\|x\|_{L_{M}}^{0} \leqq 2\|x\|_{L_{M}} \text { for all } \quad x \in L_{M}
$$

gives this constant 2 . 
The second part of the proof follows also from Theorem 3 but with a good control on the embedding norm we can prove as follows.

If $K=K(s, t) \in L_{M}\left[L_{\infty}\right]$, then $k(t)=\|K(\cdot, t)\|_{L_{\infty}} \in L_{M}$ and so

$$
\int_{T} M\left(\frac{k(t)}{\|k\|_{L_{M}}}\right) d \mu \leqq 1 .
$$

Integrating with respect to $s \in S$ and using the convexity of $M$ we obtain

$$
\begin{aligned}
& \int_{S} \int_{T} M\left(\frac{|K(s, t)|}{C\|k\|_{L_{M}}}\right) d \mu d \nu \leqq \frac{1}{C} \int_{S} \int_{T} M\left(\frac{|K(s, t)|}{\|k\|_{L_{M}}}\right) d \mu d \nu \\
\leqq & \frac{1}{C} \int_{S} \int_{T} M\left(\frac{k(t)}{\|k\|_{L_{M}}}\right) d \mu d \nu \leqq \frac{1}{C} \int_{S} d \nu=\frac{\nu(S)}{\max \{1, \nu(S)\}} \leqq 1 .
\end{aligned}
$$

Therefore, $K \in L_{M}(S \times T)$ and $\|K\| \leqq \max \{1, \nu(S)\}\|k\|_{L_{M}}$.

The second embedding we can get by duality. Since $L_{\infty}\left[L_{M^{*}}\right] \stackrel{C}{\hookrightarrow} L_{M^{*}}(S$ $\times T)$ it follows that

$$
L_{M}(S \times T)=\left(L_{M}(S \times T)\right)^{\prime} \stackrel{C}{\hookrightarrow}\left(L_{\infty}\left[L_{M^{*}}\right]\right)^{\prime}=L_{1}\left[\left(L_{M^{*}}\right)^{\prime}\right]=L_{1}\left[L_{M}\right] .
$$

The constant 2 appeared here again because the dual norm to $\|\cdot\|_{L_{M}^{*}}$ is an Orlicz norm $\|\cdot\|_{L_{M}}^{0}$ which is equivalent to $\|\cdot\|_{L_{M}}$ by (17). We can prove the last two embeddings similarly. These two last embeddings were also proved by Bukhvalov (cf. [7], Proposition 1).

\section{Operators in mixed norm spaces}

The results from Section 2 can be applied to study boundedness of some classical operators of analysis in Orlicz spaces with mixed norms. Two types of questions appear here.

The first type is to investigate boundedness of classical operators, like Hardy operator, maximal operators, integral operator or singular integral operators in mixed norm $L_{p}\left[L_{q}\right]$-spaces or their weighted versions. Such results started just after the classical paper of Benedek-Panzone [1], where the mixed norm $L_{p}\left[L_{q}\right]$-spaces appeared. 
Consider the maximal operator $M_{1}$ defined for $K(s, t)$ on $S \times R^{n}$ with a $\sigma$-finite measure $\mu$ on $S$ by

$$
M_{1} K(s, t)=\sup _{Q \ni t} \frac{1}{|Q|} \int_{Q}|K(s, y)| d y
$$

and the strong maximal operator $M_{S}$ defined by

$$
M_{S} K(s, t)=\sup _{R \ni(s, t)} \frac{1}{|R|} \int_{R}|K(x, y)| d x d y
$$

where $R=Q \times Q^{\prime}$ and $Q \in R^{m}, Q^{\prime} \in R^{n}$ are cubes. K. F. Anderssen and R. T. John (1980) proved that if $1<p, q<\infty$, then $M_{1}$ is bounded on $L_{p}(w d t)\left[l_{q}\right]$ spaces if and only if $w$ satisfies the so-called $A_{p}$-condition of Muckenhoupt: there exists a positive constant $c$ such that for all cubes $Q$

$$
\frac{1}{|Q|} \int_{Q} w(y) d y\left(\frac{1}{|Q|} \int_{Q} w(y)^{-1 /(p-1)} d y\right)^{p-1} \leqq c .
$$

The boundedness in $L_{p}(w d t)\left[L_{q}(\mu)\right]$ spaces was also proved in 1980 by V. Kokilashvili ([22], Theorem 1) (he announced it in 1978 in the Russian Doklady): Let $1<p, q<\infty$. Then $M_{1}$ is bounded on $L_{p}(w d t)\left[L_{q}(\mu)\right]$ if and only if $w$ satisfies the $A_{p}$-condition.

Kokilashvili ([22], Theorem 2) proved also the boundedness of the strong maximal operator $M_{S}$ in weighted mixed norm spaces $L_{p}(u d t)\left[L_{q}(v d s)\right]$ with separated weights: Let $1<p, q<\infty$ and let the weight $w(s, t)$ have separeted variables, that is, $w(s, t)=v(s) u(t)$. Then the strong maximal function $M_{S}$ is bounded on $L_{p}(u d t)\left[L_{q}(v d s)\right]$ if and only if $u \in A_{p}, v \in A_{q}$.

The unweighted case of this result was proved independently by Fernandez [14] in 1987. I should also mention here that the boundedness of the operators $M_{1}$ and $M_{S}$ was proved in 1984 by Bukhvalov ([8], p. 100) in weighted Orlicz spaces $L_{M}(u d t)\left[L_{N}\right]$ using the Kokilashvili results, the Kerman-Torchinsky [21] result and an interpolation technique, which we will discuss below.

Note that in the case of unseparated weight $w=w(s, t)$ the boundedness of $M_{S}$ in $L_{p}\left[L_{q}(w)\right]$ is still an open problem.

The other classical operators, like integral operators, double Hilbert transform, singular integral operators, multiplicators of Fourier integrals, Paley-Littlewood theory, Carleson-Hunt operators (theorem) can be considered in vector-valued spaces or in the mixed norm spaces $L_{p}(w d s)\left[L_{q}\right]$ (see Fernandez [14], Bukhvalov [8], Garcia-Cuerva and Rubio de Francia [18]). 
The second question is how to use the results for mixed norm $L_{p}\left[L_{q}\right]$ spaces to extend them to mixed norm Orlicz $L_{M}\left[L_{N}\right]$-spaces. The interpolation property of the Calderón-Lozanovskil construction on the class of mixed norm ideal spaces $E[F]$ with changed spaces $E$ and $F$ having the Fatou property is important but not possible except the class of $L_{p}\left[L_{q}\right]$-spaces. This follows from the Nagumo-Kolmogorov theorem for any interpolation method (cf. [5]) or in the case of Calderón-Lozanovskil construction from our Corollary 4. We can use instead of this result the interpolation in each variable independently. Note that interpolation in $E$ with any fixed space $F$ is always possible but interpolation in $F$ with fixed $E$ is not always possible, for example, it does not work for the real method of interpolation, as was observed by M. Cwikel in 1977. Bukvalov ([8], p. 98) observed that this will be possible for the Calderón-Lozanovskilu construction.

We do not need, but let us mention that the connection between various Gustavsson-Peetre's interpolation methods, Ovchinnikov's interpolation methods and the Calderón-Lozanovskii construction on vector-valued Banach ideal spaces, in particular also on mixed norm spaces with some additional properties like the Fatou property or continuous norms, can be found in [13], Theorem 3.2 (cf. [36] for definitions and general properties).

Let us apply the Bukhvalov method in the proof of the mixed norm version of the Ryan interpolation theorem. Ryan ([38], Theorem 3) proved that if $T$ is a bounded linear operator in every reflexive $L_{p}(I)$ space, then $T$ is bounded in any reflexive Orlicz space $L_{M}(I), I=[0,1]$ (cf. also [31], pp. 149150 where $I$ is either $[0,1]$ or $[0, \infty))$. We can easily see that this theorem is true for any $\sigma$-finite nonatomic measure. We will keep this assumption on the measure in the next theorem.

Theorem 4. Let $L_{M}\left[L_{N}\right]$ be a reflexive mixed norm Orlicz space. If $T$ is a linear or sublinear bounded operator on $L_{p}\left[L_{q}\right]$ for all $1<p, q<\infty$, then $T$ is defined and bounded on $L_{M}\left[L_{N}\right]$.

Proof. We shall only consider the case where $\mu(T)=\nu(S)=\infty$. The other cases can be treated analogously. Mixed norm Orlicz spaces $L_{M}\left[L_{N}\right]$ are reflexive if and only if the Orlicz spaces $L_{M}$ and $L_{N}$ are reflexive, which is also equivalent to the condition that Orlicz functions $M, N$ and their complementary functions $M^{*}, N^{*}$ all satisfy the $\Delta_{2}$-condition (cf. [31], Theorem 9.3).

Since both $M$ and $M^{*}$ satisfy the $\Delta_{2}$-condition it follows that $M(u)=$ $\varphi\left(u^{1 / p_{0}}, u^{1 / p_{1}}\right)$ for some $1<p_{0}<p_{1}<\infty$ and $\varphi \in \mathcal{U}$ (cf. [31], p. 149), and so $L_{M}=\varphi\left(L_{p_{0}}, L_{p_{1}}\right)$. Similarly since both $N$ and $N^{*}$ satisfy the $\Delta_{2}$-condition it yields that $N(u)=\psi\left(u^{1 / q_{0}}, u^{1 / q_{1}}\right)$ for some $1<q_{0}<q_{1}<\infty$ and $\psi \in \mathcal{U}$, which gives that $L_{N}=\psi\left(L_{q_{0}}, L_{q_{1}}\right)$. 
Let now $T$ be a bounded operator in $L_{p}\left[L_{q}\right]$ for any $1<p, q<\infty$. In particular, for any fixed $1<p<\infty$ the operator $T$ is bounded in $L_{p}\left[L_{q_{0}}\right]$ and $L_{p}\left[L_{q_{1}}\right]$ and by the interpolation property of the Calderón-Lozanovskiı construction on spaces with the Fatou property the operator $T$ is bounded in

$$
\psi\left(L_{p}\left[L_{q_{0}}\right], L_{p}\left[L_{q_{1}}\right]\right)=L_{p}\left[\psi\left(L_{q_{0}}, L_{q_{1}}\right)\right]=L_{p}\left[L_{N}\right]
$$

for all $1<p<\infty$. We were using here Lemma 1 . In particular, $T$ is bounded in $L_{p_{0}}\left[L_{N}\right]$ and $L_{p_{1}}\left[L_{N}\right]$, and again, by the interpolation property of the Calderón-Lozanovskil construction, $T$ is bounded in

$$
\varphi\left(L_{p_{0}}\left[L_{N}\right], L_{p_{1}}\left[L_{N}\right]\right)=\varphi\left(L_{p_{0}}, L_{p_{1}}\right)\left[L_{N}\right]=L_{M}\left[L_{N}\right]
$$

and we used here Corollary 5. The proof is complete.

Weighted version of this theorem gives the above mentioned Bukhvalov's result on the boundedness of the operators $M_{1}$ and $M_{S}$ in weighted mixed norm Orlicz spaces $L_{M}(u d t)\left[L_{N}\right]$.

\section{References}

[1] A. Benedek and R. Panzone, The space $L^{p}$, with mixed norm, Duke Math. J., 28 (1961), 301-324.

[2] J. Bergh and J. Löfström, Interpolation Spaces, Springer (Berlin, 1976).

[3] A. P. Blozinski, Multivariate rearrangements and Banach function spaces with mixed norms, Trans. Amer. Math. Soc., 263 (1981), 149-167.

[4] Yu. A. Brudnyı and N. Ya. Krugljak, Interpolation Functors and Interpolation Spaces I, North-Holland (Amsterdam, 1991).

[5] A. V. Bukhvalov, Spaces with mixed norm, Vestnik Leningrad. Univ., 19, Mat. Meh. Astronom. Vyp., 4 (1973), 5-12; English transl. in Vestn. Leningr. Univ., Math., 6 (1979), 303-311.

[6] A. V. Bukhvalov, Integral operators, and the representation of completely linear functionals on spaces with mixed norm, Sibirsk. Mat. Ž., 16 (1975), 483-493; English transl. in Siberian Math. J., 16 (1975), 368-376.

[7] A. V. Bukhvalov, Geometric applications of the Kolmogorov-Nagumo theorem, in: Qualitative and Approximate Methods for the Investigation of Operator Equations, Yaroslav. Gos. Univ. (Yaroslavl, 1983), pp. 18-33 (Russian).

[8] A. V. Bukhvalov, Theorems on interpolation of sublinear operators in spaces with mixed norm, in: Qualitative and Approximate Methods for the Investigation of Operator Equations, Yaroslav. Gos. Univ. (Yaroslavl, 1984), pp. 90-105 (Russian).

[9] A. V. Bukhvalov, Interpolation of linear operators in spaces of vector-valued functions and with mixed norm, Sib. Mat. Zh., 28 (1987), 37-51; English transl. in Sib. Math. J., 28 (1987), 24-36.

[10] A. P. Calderón, Intermediate spaces and interpolation, the complex method, Studia Math., 24 (1964), 113-190. 
[11] J. Cerdà and M. Mastyło, The type and cotype of Calderón-Lozanovskiǔ spaces, Bull. Polish Acad. Sci. Math., 47 (1999), 105-117.

[12] T. Donaldson, Inhomogeneous Orlicz-Sobolev spaces and nonlinear parabolic initial value problems, J. Differential Equations, 16 (1974), 201-256.

[13] M. Fan, On interpolation of vector-valued Banach lattices and Calderón-Lozanovskiu construction, Math. Nachr., 227 (2001), 63-80.

[14] D. L. Fernandez, Vector-valued singular integral operators on $L^{p}$-spaces with mixed norms and applications, Pacific J. Math., 129 (1987), 257-275.

[15] D. L. Fernandez, Interpolation of $2^{d}$ Banach spaces and the Calderón spaces $X(E)$, Proc. London Math. Soc., 56 (1988), 143-162.

[16] C. E. Finol and L. Maligranda, On a decomposition of some functions, Comment. Math. Prace Mat., 30 (1991), 285-291.

[17] B. Firlej and W. Matuszewska, Some remarks on spaces provided with mixed norm, Comment. Math. Prace Mat., 17 (1974), 347-357.

[18] J. Garcia-Cuerva and J. L. Rubio de Francia, Weighted Norm Inequalities and Related Topics, North-Holland (Amsterdam, 1985).

[19] A. Kamińska, L. Maligranda and L. E. Persson, Indices, convexity and concavity of Calderón-Lozanovskiǔ spaces, Math. Scand., 92 (2003), 141-160.

[20] L. V. Kantorovich and G. P. Akilov, Functional Analysis, Nauka (Moscow, 1977) (in Russian).

[21] R. A. Kerman and A. Torchinsky, Integral inequalities with weights for the Hardy maximal function, Studia Math., 71 (1982), 277-284.

[22] V. M. Kokilashvili, Maximal functions in weighted spaces, Akad. Nauk Gruzin. SSR Trudy Tbiliss. Mat. Inst. Razmadze, 65 (1980), 110-121 (in Russian).

[23] M. A. Krasnoselskiı̌ and Ya. B. Rutickiı̌, Convex Functions and Orlicz Spaces, Noordhoff (Groningen, 1961).

[24] S. G. Krein, Yu. I. Petunin and E. M. Semenov, Interpolation of Linear Operators, Nauka, Moscow, 1978 (Russian); English transl. in Amer. Math. Soc. (Providence, 1982).

[25] N. Krugljak and L. Maligranda, Calderón-Lozanovskiǔ operation on weighted Banach function lattices, J. Math. Anal. Appl., 288 (2003), 744-757.

[26] M-T. Lacroix, Comparison des espaces $E_{M}\left(\Omega_{1}, E_{M}\left(\Omega_{2}\right)\right)$ et $E_{M}\left(\Omega_{1} \times \Omega_{2}\right)$, Publ. Math. Fac. Sci. Besancon Annee, 1974-75, Exp. No. 2, 7 p. (1975).

[27] J. Lindenstrauss and L. Tzafriri, Classical Banach Spaces, II. Function Spaces, Springer-Verlag (Berlin-New York, 1979).

[28] G. Ya. Lozanovskiı̌, On some Banach lattices IV, Sibirsk. Mat. Z., 14 (1973), 140-155 (in Russian); English transl. in Siberian. Math. J., 14 (1973), 97-108.

[29] G. Ya. Lozanovskiı̌, Transformations of ideal Banach spaces by means of concave functions, in: Qualitative and Approximate Methods for the Investigation of Operator Equations, Yaroslav. Gos. Univ., Yaroslavl, 3 (1978), 122-147 (in Russian).

[30] L. Maligranda, Calderón-Lozanovskiŭ spaces and interpolation of operators, Semesterbericht Funktionalanalysis, Tübingen, 8 (1985), 83-92.

[31] L. Maligranda, Orlicz Spaces and Interpolation, Seminars in Math. 5, Univ. of Campinas (Campinas SP, Brazil, 1989).

[32] M. Milman, A note on $L(p, q)$ spaces and Orlicz spaces with mixed norms, Proc. Amer. Math. Soc., 83 (1981), 743-746.

[33] P. Nilsson, Interpolation of Banach lattices, Studia Math., 82 (1985), 135-154. 
[34] R. O'Neil, Integral transforms and tensor products on Orlicz spaces and $L(p, q)$ spaces, J. Analyse Math., 21 (1968), 1-276.

[35] V. I. Ovchinnikov, Interpolation theorems that arise from Grothendieck's inequality, Funkcional. Anal. i Priložen., 10 (1976), 45-54; English transl. in Functional Anal. Appl., 10 (1976), 287-294.

[36] V. I. Ovchinnikov, The Methods of Orbits in Interpolation Theory, Math. Reports Vol. 1, Part 2, Harwood Academic Publishers (1984), 349-516.

[37] Ja. B. Rutickiı̌, New criteria for continuity and complete continuity of integral operators in Orlicz spaces, Izv. Vyš̌. Učebn. Zaved. Mat. (1962), 87-100 (in Russian).

[38] R. Ryan, Conjugate functions in Orlicz spaces, Pacific J. Math., 13 (1963), 13711377.

(Received November 28, 2002; revised May 5, 2003)

DEPARTMENT OF MATHMATICS

LULEA UNIVERSITY OF TECHNOLOGY

SE-971 87 LULE $\AA$

SWEDEN

E-MAIL: LECH@SM.LUTH.SE 